\title{
L'ACQUISITION DE LA COMPÉTENCE PHONÉTICO-PHONOLOGIQUE : LES ACTIVITÉS DANS LES MANUELS DE FLE
}

La plupart des enseignants visent l'intelligibilité en classe (LAURET 2007 : 22), un objectif tout à fait légitime et conforme aux exigences de l'approche communicative et actionnelle. Pourtant, le manque de travail sur la compétence phonético-phonologique risque d'entraîner la fossilisation des erreurs de prononciation des apprenants. Certains auteurs parlent d'" une crise dans l'enseignement de la composante phonético-phonologique des langues étrangères aujourd'hui » (SAUVAGE, BILLIÈRES 2019).

Sachant que les enseignants se réfèrent le plus souvent au manuel, la ressource principale en classe, l'objectif de cet article serait d'analyser deux séries des manuels de FLE utilisées aux collèges en Serbie dont l'une est écrite par une auteure serbe et l'autre par différents auteurs français, selon le niveau. D'abord, nous comparons les concepts dans lesquels s'inscrivent les activités phonétiques dans les deux séries des manuels, ensuite nous classons les activités phonétiques selon la typologie donnée et finalement, nous vérifions si ces activités pourraient contribuer au développement et à l'acquisition de la compétence phonético-phonologique. Les résultats de l'analyse mettent en évidence une différence importante dans l'approche à la dimension phonéticophonologique entre deux séries des manuels étudiés.

Mots-clés : prononciation, compétence phonético-phonologique, activités, manuels, FLE

\section{Introduction}

Dans l'approche communicative et actionnelle, la phonétique est devenue " le vilain petit canard » de la didactique (BILLIĖRES 2014) ou bien « le parent pauvre » de la didactique et de l'enseignement des autres

\footnotetext{
${ }^{1}$ natasa.ignjatovic@filfak.ni.ac.rs

${ }^{2}$ Cet article est rédigé dans le cadre du projet scientifique Les langues, les littératures et les cultures romanes et slaves en contact et en divergence $\mathrm{N}^{\circ}$ 81/1-17-8-01 financé par la Faculté de Philosophie de l'Université de Niš, l'Agence universitaire de la Francophonie (AUF) et l'Ambassade de France en Serbie.
} 
compétences (DUFEU 2008, WACHS 2011, MORAZ, PRIKHODKINE 2011). Malgré le fait que la composante phonético-phonologique est inhérente à la communication, tout comme les autres compétences linguistiques (grammaticale, lexicale, sémantique, orthographique) ainsi que la compétence sociolinguistique et pragmatique, il semble qu'elle n'a pas le même statut dans les manuels, dans les pratiques enseignantes et de ce fait chez des élèves. L'approche communicative privilégie la communication, se focalisant sur la transmission et la réception du message dans son ensemble, donc comprendre et se faire comprendre, c'est-à-dire que le contenu prime sur la forme et que le travail sur la prononciation reste secondaire ou marginalisé.

«Les approches communicatives qui visent à mettre l'apprenant immédiatement au contact de la langue sous ses diverses formes, écrites en particulier, et qui incitent dès le début de l'apprentissage à la production à visée communicative, en minimisant de fait le rôle de la prononciation, tendent probablement à empêcher un développement optimal des possibilités phonétiques » (LAURET 2007: 29).

Dufeu souligne aussi que la langue écrite, étant principale en classe, rend difficile le travail sur l'oral et la prononciation (DUFEU 2008 : 2). Ceci s'explique par le fait qu'il y a un grand écart entre l'oral et l'écrit en français, car, comme on le sait très bien, à chaque phonème correspond un ou plusieurs graphèmes.

Certains auteurs considèrent que la mise à l'écart de la phonétique est due aux convictions suivantes : 1) pour développer la compétence phonéticophonologique il est suffisant d'être en contact avec la langue étrangère ; 2) on n'est pas capable d'acquérir et maîtriser la prononciation d'une langue étrangère après la puberté (l'hypothèse de période critique) ; 3) l'accent étranger ne peut pas perturber la communication (CHAMPAGNE-MUZAR, BOURDAGES 1998 : 17). Par contre, en ce qui concerne l'hypothèse des contraintes biologiques, Lauret considère que les adultes ne montrent pas nécessairement moins de succès dans la prononciation car « les études récentes ont montré que le cerveau reste flexible tout au long de la vie » (LAURET $2007: 27)$.

Pour obéir aux principes de l'approche communicative, les auteurs des manuels, comme tous les utilisateurs, se réfèrent aux recommandations données dans le CECRL et de ce fait, ils créent des manuels qui négligent la langue maternelle de futurs apprenants (sauf dans le cas où il s'agit des auteurs dont la langue maternelle est celle des apprenants). Quand il s'agit des activités phonétiques, le fait de ne pas prendre en compte le système phonologique de la langue maternelle des apprenants a comme conséquence que les activités choisies ne visent pas les difficultés des apprenants-utilisateurs du manuel. Car, les éléments phonétiques difficiles pour les apprenants ayant 
une langue maternelle commune ne sont pas nécessairement difficiles pour un autre groupe, ayant une autre langue maternelle. Ce serait idéal si les auteurs connaissaient les deux systèmes phonologiques, celui de la langue cible et celui de la langue maternelle du public auquel ils destinent le manuel. Mais, vu que le plus souvent les manuels de FLE sont destinés à un public universel, il n'est pas possible de cibler les difficultés spécifiques d'un groupe mais plutôt celles qui seraient plus ou moins communes pour les utilisateurs de n'importe quelle langue maternelle. Ceci entraîne une vision particulière de la part de l'enseignant envers la problématique de la prononciation en classe : il doit connaître les spécificités de deux systèmes phonologiques pour pouvoir sélectionner les éléments segmentaux et suprasegmentaux qui, d'après les études contrastives, posent le plus de difficultés à son public cible, s'il utilise un manuel destiné au marché international. Par contre, l'avantage d'un manuel fait par l'auteur de la même langue maternelle que le public cible, repose dans le fait que cet auteur va sélectionner les activités de prononciation connaissant les difficultés typiques des apprenants ainsi que les différences entre les deux systèmes phonologiques.

On peut estimer que la prononciation est soit très peu présente dans les manuels (LAURET 2007 : 13), soit les activités proposées ne répondent pas aux besoins du public mais aux besoins didactiques (DUFEU 2008) ou encore on trouve « une batterie d'exercices dans les manuels » (SAUVAGE, BILLIÈRES 2019 : 1). Par contre, Lauret constate que les enseignants visent l'intelligibilité mais que «l'enseignant doit toujours s'efforcer de favoriser l'acquisition maximale » (LAURET 2007 : 22). C'est-à-dire que le travail sur la prononciation devrait se faire en continuité, dès le début et tout au long de l'apprentissage en corrigeant notamment les erreurs qui se reproduisent.

Ce qui pose problème, c'est l'ambiguïté de la notion même d'intelligibilité. Car, être intelligible ne peut pas être perçu de la même manière, cela dépend de nombreux facteurs. D'abord, prenons celui qui évalue l'intelligibilité, en classe c'est l'enseignant de FLE : l'appréciation de l'intelligibilité de ses élèves par l'enseignant non natif va dépendre de ses propres habitudes perceptives et articulatoires, faisant partie du répertoire de sa langue maternelle aussi bien que de celles acquises dans la langue étrangère. Autrement dit, l'enseignant de FLE dont le serbe est la langue maternelle habitué à maitriser deux systèmes langagiers, va juger différemment l'intelligibilité d'un élève serbe parlant français et d'un élève espagnol, par exemple, parlant français, s'il n'a pas appris l'espagnol, bien sûr. Nous y voyons une raison assez pertinente à cause de laquelle l'enseignant devrait insister sur « l'acquisition maximale », comme Lauret (2007) le souligne. Car, si l'enseignant ne demande pas d'investissement de la part de ses élèves, ils ne vont pas puiser dans leurs capacités. Nous considérons aussi que le travail 
sur la prononciation, la correction de la prononciation erronée devrait faire partie des objectifs implicites dans la classe, c'est-à-dire qu'il faudrait intégrer le travail sur la compétence phonético-phonologique parallèlement avec le travail sur les autres compétences.

Étant donné que les deux séries de manuels que nous avons pris en compte pour l'analyse dans le cadre de cet article ont été éditées entre 2007 et 2012, nous nous référons au Cadre Européen commun de références pour les langues (2001) afin de consulter les prescriptions se rapportant à la compétence phonologique. Nous ne pouvons pas prendre en compte la version de CECRL (2018) car les auteurs ont dû s'appuyer sur la version existante pour concevoir les manuels, donc à la version datant de 2001, vu les dates de parution de deux séries de manuels. Les deux séries de manuels sont destinées aux élèves du collège, de la cinquième à la huitième classe, ce qui correspond aux niveaux A1 et A2 du CECRL. Donc, le CECRL (2001) définit la maitrise de la compétence phonologique comme suit :

«A1 : La prononciation d'un répertoire très limité d'expressions et de mots mémorisés est compréhensible avec quelque effort pour un locuteur natif habitué aux locuteurs du groupe linguistique de l'apprenant/utilisateur ;

A2 : La prononciation est en général suffisamment claire pour être comprise malgré un net accent étranger mais l'interlocuteur devra parfois faire répéter » (CECRL 2001 : 92).

Nous sommes obligés de mettre ici une petite parenthèse pour éclaircir un point : les échelles du CECRL se rapportant à la compétence grammaticale, lexicale, sémantique, phonologique et orthographique prévoient une progression par niveau. Mais, si on se réfère à la constatation de Lauret qui dit que « Contrairement à d'autres composantes de la langue, et même si l'on peut définir certaines priorités dans l'apprentissage de la prononciation, on n'acquiert pas les éléments phonétiques les uns après les autres comme des éléments de lexique ou de grammaire » (LAURET 2007 : 156), cela veut dire que les auteurs des manuels aussi bien que les enseignants devraient comprendre les échelles du CECRL, en ce qui concerne la compétence phonologique comme un guide car, d'abord, l'appropriation de la prononciation est plus complexe ou au moins plus exigeante, elle demande plus d'effort tant de la part de l'enseignant que de l'élève et tout utilisateur du CECRL, enseignant ou auteur doit avoir un esprit critique envers les descripteurs. Ceci dit qu'on devrait faire le travail sur la prononciation en continuité, dès le début et tout au long de l'apprentissage, tout en insistant sur une bonne prononciation afin que les apprenants soient conscients que le développement de cette compétence est aussi important que celui des autres compétences langagières.

Dans le cadre de cet article, nous allons d'abord présenter les objectifs, la méthodologie et le corpus, ensuite nous allons présenter les résultats de la recherche et nos interprétations, pour enfin parvenir aux conclusions. 


\section{Méthodologie et corpus}

En nous appuyant sur les typologies de Champagne-Muzar et Bourdages (1998), de Celce-Murcia et al. (2010) et de Lauret (2007), nous avons analysé le corpus de manuels de FLE, Le français pour nous 1-4 et Pixel 1-4 dont les concepts correspondent à l'approche communicative. Nous avons d'abord classifié les activités phonétiques se trouvant dans ces manuels selon les critères suivants :

1. les activités où l'on aborde les éléments segmentaux (les sons), les éléments suprasegmentaux (la prosodie) ou les phénomènes spécifiques ;

2. le type d'activité, selon la consigne donnée.

Ensuite, nous avons vérifié quels éléments segmentaux ou suprasegmentaux ont été pris en compte ou ont été favorisés. De plus, étant donné qu'il s'agit de séries des manuels, nous avons analysé chaque série selon les critères suivants : selon la pertinence du choix des éléments abordés dans les activités phonétiques, selon la cohérence entre les éléments phonétiques par niveau et si cela permet la progression dans l'acquisition de la compétence phonético-phonologique. Finalement, nous avons vérifié s'il y avait un écart significatif entre les éléments segmentaux et suprasegmentaux représentés dans les manuels des auteurs français et dans ceux de l'auteure serbe. Car, notre hypothèse de départ serait que les auteurs des manuels qui connaissent la langue maternelle ainsi que les difficultés typiques du public auquel ils destinent le manuel, vont aborder la composante phonologique suivant des difficultés éprouvées, à la différence des auteurs qui destinent leurs manuels au public universel. Ceci dit que le travail sur le développement de la compétence phonologique serait mieux ciblé et le choix des éléments plus approprié aux besoins des apprenants.

De plus, les approches contrastives se rapportant à la prononciation des apprenants serbophones du FLE (GUDURIĆ 2004, 2009 ; ŠOTRA 2006) nous permettent de voir que les différences entre les deux systèmes phonologiques et prosodiques sont à l'origine des difficultés de perception et production des locuteurs serbophones. Ceci concerne notamment certaines voyelles, l'accentuation et le rythme du français. D'ailleurs, on peut constater que dans la pratique, le plus souvent, les erreurs interlinguales sont dues : a) à la prononciation des voyelles qui n'existent pas dans le système vocalique serbe : la voyelle orale $[y]$, les voyelles orales à double timbre $[\mathrm{e}, \varepsilon\lrcorner,, \mathrm{o}, \propto, \varnothing]$, les voyelles nasales [õ, $\tilde{\alpha}, \tilde{\varepsilon}, \tilde{a}], b)$ ainsi qu'à la place de l'accent : en français, la dernière syllabe du mot isolé ou du groupe de sens est porteur de l'accent tandis qu'en serbe chaque mot est accentué, l'accent tombe sur la première ou deuxième syllabe. En ce qui concerne l'orthographe, vu les différences 
entre l'écriture phonétique du serbe et l'écriture étymologique du français, il serait essentiel d'enseigner parallèlement les sons avec les graphies aux élèves serbophones.

\section{Résultats de l'analyse du corpus}

La structure de deux séries des manuels étudiés est différente. Les manuels Pixel 1-4 sont composés de 7 unités (unité $0+6$ unités). Chacune des six unités contient trois leçons, une ou deux pages intitulées Civilisation, une page réservée au Bilan et dans les dernières pages des unités 2, 4 et 6 on trouve Le point sur la grammaire. Dans le contenu de chaque unité, on peut lire les objectifs visés, d'où les rubriques intitulées : Grammaire, Conjugaison, Phonétique, Thème-lexique, Objectifs de communication, Civilisation.

Les manuels Le français pour nous 1-4 sont composés de sept ou huit dossiers (cela varie selon niveau) avec des objectifs déterminés au début de chaque dossier. Les dossiers contiennent des leçons et dialogues, les parties avec le lexique issu de la leçon avec sa traduction en serbe, les énoncés communicatifs mis en évidence ainsi que les points de grammaire étudiés.

L'alphabet phonétique dans Pixel 1-4, se trouvant à la dernière page de chaque cahier d'exercices représente les phonèmes de la langue française :

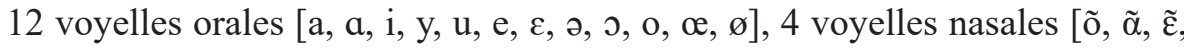
ã], 3 semi-voyelles [w, j, u], et 19 consonnes : ici on note les 17 consonnes du français standard [p, t, k, b, d, g, f, s, J, v, z, z, l, R, m, n, n], une consonne vélaire nasale empruntée à l'anglais [n] (camping); mais ce qui étonne c'est la représentation de $[\mathrm{nj}]$ comme phonème avec la graphie $n i$ et l'exemple donne : panier. Ici, il s'agit plutôt de la consonne [n] + semi-voyelle [j].

Dans les manuels Le français pour nous 1-4 on ne trouve pas d'alphabet phonétique représenté séparément dans une page, mais on y trouve les activités en lien avec les 4 nasales, 2 semi voyelles [w, j], 10 voyelles orales $[i, y, u, e, \varepsilon, \partial, \rho, o, \propto, \varnothing]$ et 9 consonnes [1, R, k, g, s, z, $\left.\int, 3, f\right]$, ce qui est dû au fait que l'approche à la dimension phonético-phonologique est complètement différente par rapport à celle des auteurs de Pixel 1-4. En effet, dans Le français pour nous 1-4 les activités phonétiques visant les phonèmes sont plutôt mises en relation avec l'acquisition des graphèmes correspondants. C'est-à-dire que, d'un côté, l'auteure propose les activités phonétiques en les mettant en relation avec les graphies correspondantes, sachant que la langue serbe possède une écriture phonétique - à chaque phonème correspond un graphème, à la différence de l'orthographe étymologique de la langue française - à un phonème correspondent un ou plusieurs graphèmes, et de l'autre côté, elle vise les difficultés dues aux différences entre les systèmes prosodiques de 
deux langues, en proposant les activités de répétition et de lecture de chaque dialogue, intégrées dans l'apprentissage.

Dans Pixel 1, on trouve des activités qui sont orientées vers l'acquisition des segments : les voyelles orales $[y],[u],[e],[\varepsilon],[\ni],[œ],[\varnothing]$, les voyelles

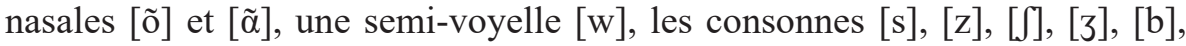
$[\mathrm{v}],[\mathrm{R}]$ tandis que parmi les éléments suprasegmentaux on trouve une activité qui vise l'appropriation du rythme. Quant aux phénomènes spécifiques, on trouve la liaison avec le son [z]. Selon les consignes données, il s'agit des activités d'écoute suivies par repérage, répétition/imitation, identification, discrimination et chant, le plus souvent, ou plus rarement il s'agit des activités d'écoute suivies par répétition ou lecture. On ne trouve qu'une activité de production dirigée dans le livre de l'élève et deux dictées à trous dans le cahier d'exercices, en ce qui concerne la relation entre le code oral et le code écrit. Les contenus qui visent le travail sur la compétence phonéticophonologique se trouvant dans les manuels sont suivis par les activités dans le cahier relatives aux mêmes objectifs phonético-phonologiques. C'est-à-dire qu'il y a une cohérence et que l'élève peut travailler de façon autonome sur les contenus acquis en classe. Ceci obéit à l'un des principes de l'approche communicative et actionnelle, favoriser l'autonomie de l'apprenant. Dans ce manuel, nous estimons la présence des chansons qui, par leur aspect ludique et amusant peuvent fortement contribuer à l'acquisition des sons visés : les mots de ces chansons, contenant les sons étudiés, développent la perception auditive et de ce fait incitent la discrimination des sons étudiés, par exemple $[\mathrm{e}] /[\varepsilon]$ : «Je vais à la fête de la belle Alizée, il y a aussi Juliette, on va bien danser » (FAVRET $2011: 25$ ). Il en est de même pour les virelangues que l'on trouve dans ce manuel et qui permettent non seulement l'identification des sons étudiés mais aussi la création d'une bonne ambiance en classe qu'apporte ce type d'activité. C'est le cas, par exemple, avec un virelangue chanté ayant les sons $\left.\left[\int\right] /[3]\right)$ : «Un jeune chat jaune achète du chou rouge pour son chaton » (FAVRET 2011:35).

Le manuel Pixel 2 contient les activités phonétiques dans lesquelles on trouve les mêmes sons comme dans Pixel 1 : les voyelles orales : [y], [u], [e], $[\varepsilon],[ə]$ (on note pourtant l'absence des sons [œ] et [ø] par rapport au livre précédent) et une semi-voyelle [w] (la même que dans le livre du niveau 1).

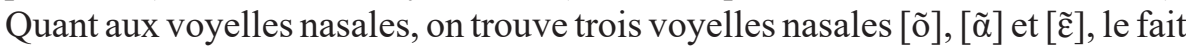
que cette dernière a été rajoutée dans ce manuel laisse supposer que les auteurs ont voulu introduire progressivement le travail sur les voyelles nasales. Les auteurs proposent également les graphies pour ces voyelles nasales : $[\tilde{\alpha}]-$ an, en; [ $\tilde{o}]$ - on; [ $[\tilde{\varepsilon}]$ - in, ain, ein, un. On voit bien que la dernière graphie est celle

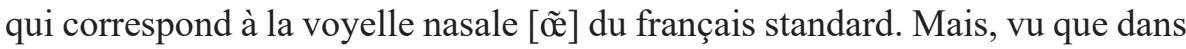

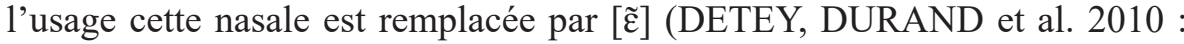


151 ; GUDURIĆ 2009 : 118-119 ; LÉON 1966 : 39), ce choix de l'auteur est compréhensible. Néanmoins, dans Pixel 3 on trouve une activité où les quatre voyelles nasales sont représentées : on demande aux élèves d'écouter, repérer et classer les mots selon la nasale que les mots entendus contiennent. Cela représente une incohérence entre ces deux manuels, et de plus, cela peut provoquer des confusions chez les élèves ou même chez l'enseignant. Quant aux consonnes, on note la présence des activités qui visent la discrimination des consonnes $[\mathrm{b}] /[\mathrm{v}],[\mathrm{v}] /[\mathrm{f}]$ ainsi que l'identification des occlusives sourdes et sonores [p], [t], [k], [b], [d], [g]. Quant aux éléments suprasegmentaux, on trouve les activités qui visent l'intonation ainsi qu'une activité sur la liaison avec le son [t] : il s'agit des phrases interrogatives à l'inversion : "Fait-il du sport ? Doit-il faire du sport ? Prend-il le bus ? (SCHMITT 2013 : 37). Mais, on constate aussi, comme c'est le cas avec Pixel 1, une prédominance des activités qui visent l'acquisition des sons. Dans le cahier d'exercices qui accompagne Pixel 2, on trouve moins d'activités phonétiques que dans Pixel 1. Dans Pixel 2 il s'agit du même type d'activités : écoute, repérage, répétition/ imitation/chant, identification/discrimination. Il n'y a pas d'activités qui visent la relation phonie-graphie. On trouve une activité de lecture qui vise l'acquisition de la liaison avec le son [t].

Dans Pixel 3, les activités phonétiques sont précédées par un petit titre Comprends et prononce où l'on propose des activités sur les voyelles orales $[\mathrm{y}] /[\mathrm{u}]$ et $[\mathrm{\jmath}] /[\mathrm{e}]$ (pourtant il n'y a pas de voyelle $[\varepsilon]$ ), on trouve une activité

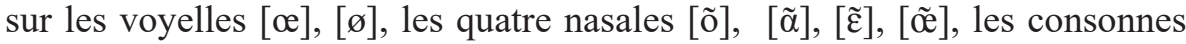
$[\mathrm{v}],[\mathrm{b}],[\mathrm{f}],[\mathrm{C}],[3],[\mathrm{z}],[\mathrm{s}]$. On introduit ici, pour la première fois dans cette collection de manuels, des activités portant sur les semi-voyelles [u] et [j]. Quant aux éléments suprasegmentaux, on trouve des activités sur le rythme et l'intonation. En ce qui concerne les phénomènes spécifiques, les auteurs proposent des activités de liaison avec les sons [t] et [z]. Ce qui différencie ce manuel par rapport aux deux manuels précédents de cette série, est d'abord le fait qu'il n'y a pas d'activités phonétiques dans le cahier respectif, ensuite, la plupart de toutes les activités phonétiques est liée à l'acquisition des éléments morpho-phonétiques : par exemple, le genre de noms en [̃̃]/[on] : le champion/la championne. On note aussi les activités phonétiques qui servent à la fois à l'appropriation du lexique, comme c'est le cas avec les adjectifs qui servent à caractériser quelqu'un : au masculin en [œ] dans la syllabe finale fermée (travailleur), au masculin dans la syllabe ouverte en [ø] (amoureux) et la prononciation de la voyelle fermée [ø] au féminin dans la syllabe fermée devant [z] (amoureuse). Il s'agit toujours du même type d'activités : repérage, répétition/imitation, identification, discrimination, précédées par l'écoute. Par exemple, dans une activité de discrimination on propose aux élèves de distinguer les sons dans les paires minimales : «[ə]/[e] : Je voudrais ce livre 
/ ces livres ; [ui]/[ui] : Oui, c'est lui, c'est Louis ; [y]/[u] : Tu es sûr / sourd ; $[\mathrm{z}] /[\mathrm{s}]$ : Au menu, il y a du poisson / poison ; [j]/[3] : Tourne la page /la paille »

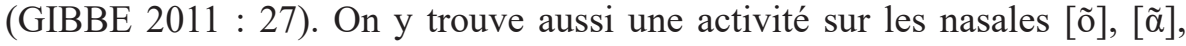
$[\tilde{\varepsilon}],[\tilde{\varrho}]$ où l'on met en relation les sons avec leurs graphies correspondantes. Quant au rythme et à l'intonation, les auteurs proposent une activité qui vise la perception du rythme des verbes au futur simple et la non prononciation du [ə] caduc : « Je donnerai, on échangera, tu partageras; on prêtera...» (GIBBE 2011 : 33) ainsi qu'une activité visant le repérage de l'intonation montante/ descendante. Pour travailler sur la liaison avec les sons $[\mathrm{t}]$ et $[\mathrm{z}]$, on trouve les activités de repérage/répétition et de lecture.

Dans Pixel 4, on peut voir des activités qui visent les segments : les voyelles orales $[\mathrm{u}] /[\mathrm{y}],[\mathrm{e}] /[\varepsilon]$; les voyelles nasales $[\tilde{o}],[\tilde{\alpha}],[\tilde{\varepsilon}]$ (on note ici encore l'incohérence concernant le choix des nasales qui varie selon les auteurs de cette série des manuels); les consonnes : [b]/[v], [d], [t], [p], [b]. Quant aux éléments suprasegmentaux, on trouve des activités sur l'intonation en lien avec les sentiments (doute, satisfaction, colère, déception, etc.) ainsi que sur le rythme : le rythme et les rimes (plate, par deux, croisée). Dans le cahier d'exercices, on présente les mêmes éléments phonétiques comme dans le livre de l'élève. À part les activités d'écoute, repérage/identification, discrimination, répétition/imitation/lecture, dans ce manuel on trouve aussi des activités de production dirigée (une strophe en rime) ainsi que des activités qui relient les phonies avec les graphies (les mots/les phrases à trous avec graphies proposées), comme par exemple «Écoute et complète avec an ou on » : a) Hier, j'ai m....gé du th.... avec de la soupe au potir... . En dessert, j'ai pris un gr....d macar.... . » (COUDERC 2013 : 41).

Dans les manuels Le français pour nous 1-4, on trouve une différente approche de la prononciation et de la dimension phonético-phonologique et orthographique par rapport à celle de la série des manuels Pixel 1-4. Ce manuel étant fait par une spécialiste serbe, qui connaît les systèmes phonologiques du français et du serbe, d'après la structure des manuels proposée, vise les objectifs phonético-phonologiques en mettant en relation les sons et les graphies. Ceci s'explique aussi par le fait que l'auteure essaie de faire progresser le travail sur l'acquisition de la prononciation, dans une approche que nous osons appeler « intégrative». Car, elle vise le travail sur la prononciation parallèlement avec les autres compétences : on trouve les activités de compréhension précédées par le travail sur la prononciation. Sachant qu'en serbe l'accent tombe sur la première ou deuxième syllabe du mot, tandis qu'en français c'est sur la dernière syllabe du mot isolé ou du groupe de sens et que, de ce fait, ce type d'erreurs est très fréquent dans l'interlangue des apprenants serbes, l'auteure a insisté sur les activités de répétition des énoncés entiers. De plus, les activités de répétition des énoncés peuvent servir à introduire un travail plus ciblé, cela 
aide l'enseignant à repérer plus facilement les erreurs des élèves, pour pouvoir ensuite y remédier et procéder à la correction individuelle.

Dans le manuel Le français pour nous 1 , des leçons et dialogues sont précédés par les consignes «écoutez, répétez, lisez » et dans le cahier d'activités on trouve de courts énoncés communicatifs issus de leçons avec ces mêmes consignes. Quant aux activités qui ne sont pas intégrées, on trouve celles qui relient les phonèmes et les graphèmes : dans de petites sections intitulées Comment lire ? on trouve les mots avec les graphies en rouge correspondant aux phonèmes étudiés. L'auteure a visé l'appropriation de la prononciation des sons avec leurs réalisations graphiques en choisissant les sons et leurs graphies en fonction de leur fréquence dans les leçons étudiées. On trouve dans ce manuel les graphies avec les phonèmes correspondants comme suit : qu, c [k], ou [u], ai /E/, au /O/, f, ph [f], g, j [3], i, y [i], oi [wa], eu [œ], eau

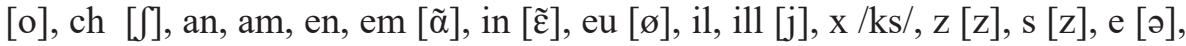

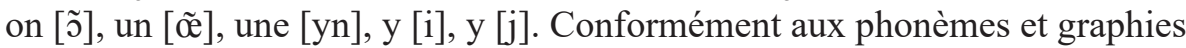
étudiés, les activités de repérages et identification des sons et des graphies se trouvent dans le cahier d'activités qui accompagne ce manuel.

Des leçons et dialogues se trouvant dans le manuel Le français pour nous 2 sont aussi imprégnés par les activités d'écoute, répétition et lecture mais on n'y trouve pas d'activités visant les objectifs phonétiques qui accompagnent ces leçons. Par contre, dans le cahier d'activités de ce manuel on trouve non seulement de courts énoncés communicatifs visant l'écoute et la répétition mais aussi des activités de repérage, identification et lecture, d'activités intitulées Comment lire? Comment écrire? qui relient les sons et les graphies. Il s'agit des activités avec des supports écrits, mais elles ne sont pas accompagnées de supports audio. L'objectif principal de ces activités serait l'appropriation des graphies mises en relation avec les phonèmes correspondants: $[\mathrm{e}],[\varepsilon],[ə] ;[\mathrm{u}]$;

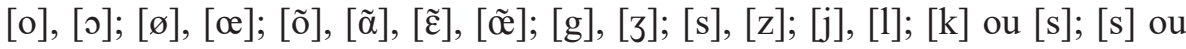
[z]; [j] ou [1]; [g], [3] ou [z]; [k] + [s]; [s] ou [z]. Plus précisément, on propose les symboles phonétiques ainsi que les mots où les graphies correspondantes sont mises en gras: [0] : robe, globe, mode, épisode, comme, somme, donne, porte, etc. ; [o] : mot, pot, sot, rose, close, pose, côte, tôt, eau, beau, tableau, chapeau, chaud, faut, faux, etc. et puis on propose un petit texte à lire. À la page suivante on donne les graphies pour le son /o/- o, eau, au, avec les mots qui contiennent ce son et à la fin, un petit texte à recopier, ayant les mots avec ces graphies (TOČANAC-MILIVOJEV 2008 : 31-32). On y trouve aussi

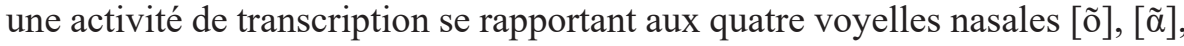

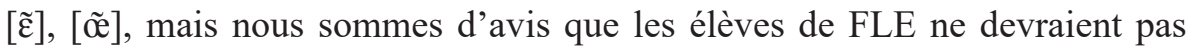
apprendre à transcrire.

Dans Le français pour nous 3, on trouve des activités d'écoute, d'imitation, de répétition, de lecture de dialogues et des leçons. Toutes ces 
activités sont aussi intégrées dans l'apprentissage et il n'y a pas d'activités phonétiques complémentaires. Dans le cahier d'activités on trouve des activités d'écoute, de répétition et de lecture dont les consignes contiennent les prescriptifs qu'il faut faire attention aux sons et à l'intonation : «Lisez le texte, répétez phrase par phrase en faisant attention à la prononciation des sons et à l'intonation » (TOČANAC-MILIVOJEV 2009: 30).

Le dernier manuel de cette série, Le français pour nous 4, suit le même concept que celui du manuel précédent, Le français pour nous 3 : les activités d'écoute, d'imitation, de répétition et de lecture sont intégrées dans l'apprentissage. Dans le cahier respectif on trouve aussi le même type d'activités, les phrases qui portent sur la prononciation de certains segments et/ou intonations, ayant comme consigne: «Écoutez et répétez correctement » (TOČANAC-MILIVOJEV 2010). Étant donné que les cahiers d'exercices sont destinés à un travail autonome à la maison, nous considérons que ces consignes ne suffisent pas et que cela ne garantit pas une autocorrection réussie par l'élève lui-même. Mais, si ces activités sont abordées en classe, l'enseignant pourra remédier aux erreurs du niveau segmental et suprasegmental pour ensuite corriger individuellement ses élèves.

\section{Conclusion}

Après avoir étudié et comparé les deux séries de manuels du corpus, nous pouvons constater que l'approche du développement de la compétence phonético-phonologique des auteurs français et de l'auteure serbe est différente. Les concepts dans lesquels les activités phonétiques sont inscrites nous permettent de soutenir cette affirmation.

Dans la série des manuels Pixel 1-4 on trouve les activités phonétiques proposées dans chaque unité, visant le plus souvent les éléments segmentaux tandis que les éléments suprasegmentaux et les phénomènes spécifiques sont pris en compte plus rarement. On note aussi, selon le type d'activités, supports et consignes données, une uniformité dans les quatre livres de l'élève de Pixel. On peut dire que, le plus souvent, les activités visant la perception auditive et la prononciation sont organisées autour des consignes écoutez, répétez, imitez, chantez, identifiez, discriminez, lisez tandis qu'il y a moins d'activités de production écrite qui demandent d'établir le lien entre les phonèmes et les graphèmes. Pourtant, en ce qui concerne le choix des éléments phonétiques représentés dans ces activités on peut voir que cela varie selon l'auteur, ce qui entraîne parfois un manque de cohérence entre les manuels de la dite série. De plus, les activités proposées ne prennent pas en compte la langue maternelle de l'apprenant mais visent plutôt les difficultés générales qui peuvent apparaître chez les apprenants. 
La série des manuels Le français pour nous 1-4 fait preuve d'une approche « intégrative » où le travail sur la dimension phonético-phonologique se fait parallèlement avec les autres compétences. Les leçons et dialogues présentés dans les quatre manuels sont précédés par les consignes écoutez, imitez, répétez, lisez, ce qui suggère à l'enseignant d'intégrer le travail sur la prononciation en classe. C'est le principe que l'auteure suit dans les quatre manuels. On trouve les activités qui visent l'acquisition des phonèmes en relation avec les graphèmes dans le manuel et cahier d'activités du niveau 1, puis dans le cahier d'activité du niveau 2. Les cahiers d'activité des niveaux 1 et 2 contiennent aussi de courts énoncés communicatifs précédés par les consignes écoutez, répétez, lisez. Les cahiers d'activités des niveaux 3 et 4 obéissent d'une manière cohérente à l'approche mise en place par l'auteure. C'est-à-dire qu'on y trouve également des activités visant un travail sur les éléments segmentaux et suprasegmentaux. On voit que l'auteure, connaissant les difficultés des élèves dont le serbe est la langue maternelle et en prenant en compte les différences entre les systèmes phonologiques de deux langues, offre dès le début de l'apprentissage, des activités visant l'acquisition des graphèmes, d'une manière systématique et cohérente qui permet aux élèves de retenir les graphèmes les plus fréquents qui correspondent aux phonèmes étudiés.

Tout compte fait, même s'il s'agit de deux séries de manuels qui sont conformes à l'approche communicative, l'approche de la dimension phonético-phonologique varie selon l'auteur. On le voit dans le choix des activités, des consignes et démarches proposées ainsi que dans la place accordée à la dimension phonético-phonologique dans les manuels. Ceci rappelle le besoin de faire des recherches dans ce domaine afin d'aboutir aux résultats qui offriraient des pistes moins ambiguës, tant aux auteurs des manuels qu'aux enseignants de FLE, pour pouvoir ensuite intégrer le travail sur la dimension phonético-phonologique en classe. D'ailleurs, il faut absolument souligner qu'une nouvelle version du CECRL (2018) a été élaborée et mise en place récemment. Si l'on se réfère aux niveaux A1 et A2 pris en compte dans notre étude, nous voyons que les nouveaux descripteurs on été complétés en précisant ce que l'apprenant devrait atteindre au niveau segmental («articulation des sons ») et au niveau suprasegmental («traits prosodiques »). On peut voir que le mot clé est « la coopération» de la part de l'interlocuteur et que l'on attend de l'apprenant qu'il soit intelligible, aidé par son interlocuteur, malgré l'influence de l'accent, du rythme et de l'intonation des autre langues parlées par l'apprenant - niveau A2 (CECRL 2018 : 142). Ceci est important pour évaluer l'élève, car l'enseignant doit être conscient de ce qu'un apprenant devrait atteindre, c'est « l'intelligibilité », au sens large du terme. Nous estimons que ce renouvellement des échelles provient, entre 
autres, du besoin de rendre plus clair et plus précis le fait que l'acquisition de la compétence phonologique est plus complexe que les autres compétences langagières.

Pour les utilisateurs des manuels, surtout pour les enseignants, il serait essentiel de choisir les activités phonétiques parmi celles figurant dans le manuel utilisé, en fonction des difficultés de prononciation des élèves. Comme on a pu constater plus haut, les difficultés communes pour les apprenants serbophones sont dues aux différences entre les systèmes vocaliques (les voyelles à double timbre et les voyelles nasales du français) ainsi qu'à la prosodie (l'accentuation et le rythme), mais il faudrait aussi diagnostiquer les erreurs individuelles des apprenants avant de procéder à la correction. En ce qui concerne l'acquisition des graphèmes, il faudrait les faire repérer systématiquement dans les mots, surtout les plus fréquents, pour ensuite proposer les activités de leur fixation.

\section{Bibliographie}

BILLIÈRES 2014 : BILLIÈRES, Michel. La phonétique, vilain petit canard de la didactique. 2014. <https://www.verbotonale-phonetique.com/ phonetique-didactique $>$.

CELCE-MURCIA, BRINTON et al. 2010 : CELCE-MURCIA, Marianne et Donna M. BRINTON, Janet M. GOODWIN with Barry GRINER. Teaching prononciation: a course book and reference guide, seconde edition. New York : Cambridge University Press, 2010.

CHAMPAGNE-MUZAR, BOURDAGES 1998 : CHAMPAGNE-MUZAR, Cécile et Johannes S. BOURDAGES. Le point sur la phonétique. Paris : CLÉ International, 1998.

CECRL 2001 : Conseil de l'Europe. Cadre européen commun de référence pour les langues : apprendre, enseigner, évaluer. 2001. <https://rm.coe. int/16802fc3a $8>$

CECRL VOLUME COMPLEMENTAIRE 2018 : Conseil de l'Europe. Cadre européen commun de référence pour les langues : apprendre, enseigner, évaluer. Volume complémentaire avec de nouveaux descripteurs. 2018. $<$ https://rm.coe.int/cecr-volume-complementaire-avec-de-nouveauxdescripteurs/16807875d5>.

DETEY, DURAND et al. 2010 : DETEY, Sylvain et Jacques DURAND, Bertrand LAKS et Chantal LYCHE. Les variétés du français parlé dans l'espace francophone, ressources pour l'enseignement. Paris : Ophrys, 2010.

DUFEU 2008 : DUFEU, Bernard. L'importance de la prononciation dans l'apprentissage d'une langue étrangère. 2008. <http://www.francparleroif.org/images/stories/dossiers/phonetique_dufeu.htm $>$ 
GUDURIĆ 2004 : GUDURIĆ, Snežana. O prirodi glasova. Beograd : Zavod za udžbenike i nastavna sredstva, 2004.

GUDURIĆ 2009: GUDURIĆ, Snežana. Osnovi fonetike s fonologijom francuskog jezika. Beograd : Zavod za udžbenike i nastavna sredstva, 2009.

LAURET 2007 : LAURET, Bertrand. Enseigner la prononciation du français : questions et outils. Paris : Hachette, 2007.

LÉON 1966 : LÉON, Pierre. Prononciation du français standard. Paris : Librairie Marcel Didier, 1966.

MORAZ, PRIKHODKINE 2011 : MORAZ, Myriam et Alexei PRIKHODKINE. « Phonétique et approche actionnelle : une mise en pratique ». A contrario 15 (2011) : 99-116.

POLOVINA 2002 : POLOVINA, Pera. Francuski pravopis, sa vežbama u laboratoriji $i$ slušaonici. Beograd : Zavod za udžbenike i nastavna sredstva, 2002.

RADUSIN-BARDIĆ 2019 : RADUSIN-BARDIĆ, Nataša. « Fonetske vežbe u savremenim metodama za učenje francuskog kao stranog jezika ». Jezici i kulture u vremenu i prostoru VIII/2 (2019) : 507-518.

SAUVAGE, BILLIÈRES 2019 : SAUVAGE Jérémi et Michel BILLIÈRES. «Enseigner la phonétique d'une langue étrangère : bilan et perspectives. » Recherches en didactique des langues et des cultures 16-1 (2019) < http://journals.openedition.org/rdlc/4234>

ŠOTRA 2006 : ŠOTRA, Tatjana. Kako progovoriti na stranom jeziku. Beograd : Zavod za udžbenike i nastavna sredstva, 2006.

ŠOTRA 2010 : ŠOTRA, Tatjana. Didaktika francuskog kao stranog jezika. Beograd : Filološki fakultet, 2010.

TOČANAC-MILIVOJEV 1997 : TOČANAC-MILIVOJEV, Dušanka. Metode u nastavi i učenju stranog jezika. Beograd : Zavod za udžbenike i nastavna sredstva, 1997.

WACHS 2011 : WACHS, Sandrine. « Tendances actuelles en enseignement de la prononciation du français, langue étrangère (FLE) ». Revista de Lenguas Modernas $N^{0} 14$ (2011) : 183-196.

\section{Corpus pédagogique}

FAVRET 2011 : FAVRET, Catherine. Pixel 1, méthode de français. Paris : CLÉ International, 2011.

FAVRET, SCHMITT 2011 : FAVRET, Catherine et Sylvie SCHMITT. Pixel 1, cahier d'exercices. Paris : CLÉ International, 2011.

SCHMITT 2011 : SCHMITT, Sylvie. Pixel 2, méthode de français. Paris : CLÉ International, 2011.

CALlet 2011 : CALLET, Stéphanie. Pixel 2, cahier d'exercices. Paris : CLÉ International, 2011. 
GIBBE 2011 : GIBBE, Colette. Pixel 3, méthode de français. Paris : CLÉ International, 2011.

ROBERT 2011 : ROBERT, Patricia. Pixel 3, cahier d'exercices. Paris : CLÉ International, 2011.

SCHMITT, Sylvie. Pixel 4, méthode de français. Paris: CLÉ International, 2012.

COUDERC 2012 : COUDERC, Anne-Cécile. Pixel 4, cahier d'exercices. Paris : CLÉ International, 2012.

TOČANAC-MILIVOJEV 2007 : TOČANAC-MILIVOJEV, Dušanka. Le français pour nous 1, francuski jezik za peti razred osnovne škole, prva godina učenja. Beograd : Zavod za udžbenike, 2007.

TOČANAC-MILIVOJEV 2007 : TOČANAC-MILIVOJEV, Dušanka. Le français pour nous 1, francuski jezik za peti razred osnovne škole, prva godina učenja, radna sveska. Beograd : Zavod za udžbenike, 2007.

TOČANAC-MILIVOJEV 2008 : TOČANAC-MILIVOJEV, Dušanka. Le français pour nous 2, francuski jezik za šesti razred osnovne škole, druga godina učenja. Beograd : Zavod za udžbenike, 2008.

TOČANAC-MILIVOJEV 2008 : TOČANAC-MILIVOJEV, Dušanka. Le français pour nous 2, francuski jezik za šesti razred osnovne škole, druga godina učenja, radna sveska. Beograd : Zavod za udžbenike, 2008.

TOČANAC-MILIVOJEV 2009 : TOČANAC-MILIVOJEV, Dušanka. Le français pour nous 3, francuski jezik za sedmi razred osnovne škole, treća godina učenja. Beograd : Zavod za udžbenike, 2009.

TOČANAC-MILIVOJEV 2009 : TOČANAC-MILIVOJEV, Dušanka. Le français pour nous 3, francuski jezik za sedmi razred osnovne škole, treća godina učenja, radna sveska. Beograd : Zavod za udžbenike, 2009.

TOČANAC-MILIVOJEV 2010 : TOČANAC-MILIVOJEV, Dušanka. Le français pour nous 4, francuski jezik za osmi razred osnovne škole, četvrta godina učenja. Beograd : Zavod za udžbenike, 2010.

TOČANAC-MILIVOJEV 2010 : TOČANAC-MILIVOJEV, Dušanka. Le français pour nous 4, francuski jezik za osmi razred osnovne škole, četvrta godina učenja, radna sveska. Beograd : Zavod za udžbenike, 2010. 
Наташа В. Игњатовић

\section{УСВАЈАҢЕ ФОНЕТСКО-ФОНОЛОШКЕ КОМПЕТЕНЦИЈЕ: АКТИВНОСТИ У УЏБЕНИЦИМА ЗА ФРАНЦУСКИ ЈЕЗИК КАО СТРАНИ}

Већина наставника поставља као циљ разумљивост на часу (LAURET 2007 : 22), што је легитимно и у складу са захтевима комуникативног и акционог приступа. Ипак, недостатак рада на фонетско-фонолошкој компетенцији може да допринесе фосилизацији грешака у изговору ученика. Неки аутори говоре о „кризи у настави фонетско-фонолошке компетенције страних језика данас" (SAUVAGE, BILLIÈRES 2019).

Знајући да наставници најчешће прибегавају коришћењу уџбеника као главном средству за рад, циљ овог чланка је да анализира две серије уџбеника за француски језик као страни који се користе у основним школама у Србији, од којих је једну написала српска ауторка док другу потписују различити француски аутори, у зависности од нивоа.

Најпре упоређујемо сам концепт фонетских активности унутар двеју серија уџбеника, затим класификујемо фонетске активности на основу дате типологије и најзад, проверавамо да ли би ове активности могле да допринесу развијању и усвајању фонетско-фонолошке компетенције. Резултати анализе указују на значајну разлику у приступу фонетско-фонолошкој димензији између две серије посматраних уџбеника.

Кључне речи: изговор, фонетско-фонолошка компетенција, активности, уџбеници, француски језик као страни 